\title{
Luminescent Device for the Detection of Oxidative Stress Biomarkers in Artificial Urine
}

\author{
Gopal Ammanath, ${ }^{\dagger, \uparrow, \S}$ Umit Hakan Yildiz, Alagappan Palaniappan, ${ }^{*, \dagger, \S}$ and Bo Liedberg ${ }^{*}, \dagger, \uparrow, \S_{(0)}$ \\ ${ }^{\dagger}$ Centre for Biomimetic Sensor Science, Nanyang Technological University, Singapore 637553 \\ ${ }^{\S}$ Nanyang Institute of Technology in Health and Medicine, Interdisciplinary Graduate School, Nanyang Technological University, \\ Singapore 637553 \\ ${ }^{\ddagger}$ School of Materials Science and Engineering, Nanyang Technological University, Singapore 639798 \\ "Department of Chemistry, Izmir Institute of Technology, Urla, 35430 Izmir, Turkey
}

\section{Supporting Information}

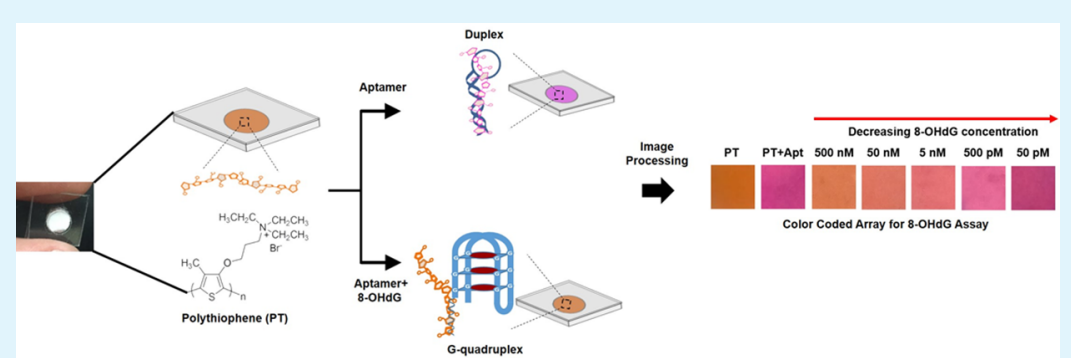

ABSTRACT: A luminescent paper-based device for the visual detection of oxidative stress biomarkers is reported. The device consists of a polyvinylidene fluoride membrane impregnated with poly(3-alkoxy-4-methylthiophene) (PT) for colorimetric detection. 8-hydroxy-2'-deoxyguanosine (8-OHdG), a biomarker associated with oxidative stress, is used as a model system for validating the proposed methodology. The detection strategy is based on monitoring the changes in optical properties of PT associated with its conformational changes upon interaction with an aptamer in the presence and in the absence of 8-OHdG. Fluorometric and colorimetric monitoring revealed linear responses for 8-OHdG concentrations between $50 \mathrm{pM}$ and $500 \mathrm{nM}$ $(\sim 14 \mathrm{pg} / \mathrm{mL}$ to $140 \mathrm{ng} / \mathrm{mL})$, with limits of detection of $\sim 300 \mathrm{pM}$ and $\sim 350 \mathrm{pM}$, respectively for $(n=3)$. Colorimetric responses in artificial urine ascertained rapid, sensitive, and selective detection of 8-OHdG at clinically relevant (pM to $\mathrm{nM}$ ) concentration levels. Furthermore, the proposed methodology enables point-of-care diagnostics for oxidative stress without requiring sophisticated instrumentation.

KEYWORDS: paper-based biosensor, naked eye detection, polythiophene, artificial urine, oxidative stress, 8-OHdG

\section{INTRODUCTION}

Reactive oxygen species (ROS) are chemically active oxygencontaining molecules that are produced as byproducts of various physiological, metabolic, and biochemical processes. While low levels of ROS aid cell signal transduction and immune response, an increase in their steady-state levels causes oxidative stress. ${ }^{1,2}$ Subsequent imbalance in oxidants and antioxidants in the normal redox state of the cells produces peroxides and free radicals that damage proteins, lipids, carbohydrates, and nucleic acids. ${ }^{3,4}$ The damage in these molecules causes cell death and progresses to various diseases such as cancer, neurodegenerative disorders, cardiovascular disease, inflammatory disease, and diabetes mellitus. ${ }^{5,6}$ The elevated oxidative stress levels is, therefore, a precursor state in these diseases. ${ }^{5}$ Thus, the detection of oxidative stress-related biomarkers could assist in the early diagnosis as well as in monitoring of disease progression.

ROS-induced oxidation results in the formation of numerous DNA fragments. Among them, 8-hydroxy-2'-deoxyguanosine (8-OHdG) is a repaired product of oxidative guanine lesions and an important biomarker for oxidative stress. Thus, the assay of 8 -OHdG helps in the early detection of diseases such as diabetes, cancer, and neurodegenerative disorders. ${ }^{7,8} 8$-OHdG has been quantified in various biological samples such as urine, saliva, blood, and tissue. ${ }^{9-12}$ Reports indicate that the average levels of 8 -OHdG are $\sim 20$ and $\sim 80 \mathrm{ng} / \mathrm{mL}$ in healthy and diseased subjects, respectively, thereby requiring sensitive methodologies for their assay. ${ }^{11,13}$

The most commonly used analytical techniques for the detection of $8-\mathrm{OHdG}$ in urine are high-performance liquid chromatography-electrochemical detection (HPLC-ECD), ${ }^{14}$ HPLC tandem mass spectrometry (HPLC MS/MS), ${ }^{15}$ capillary electrophoresis with electrochemical detection (CEECD), ${ }^{12,16}$ and electrochemical methods including cyclic voltammetry $(\mathrm{CV}),{ }^{17-19}$ differential pulse voltammetry $(\mathrm{DPV}),{ }^{20}$ and linear sweep voltammetry (LSV). ${ }^{21}$ Ultraviolet

Received: November 13, 2017

Accepted: February 12, 2018

Published: February 12, 2018 


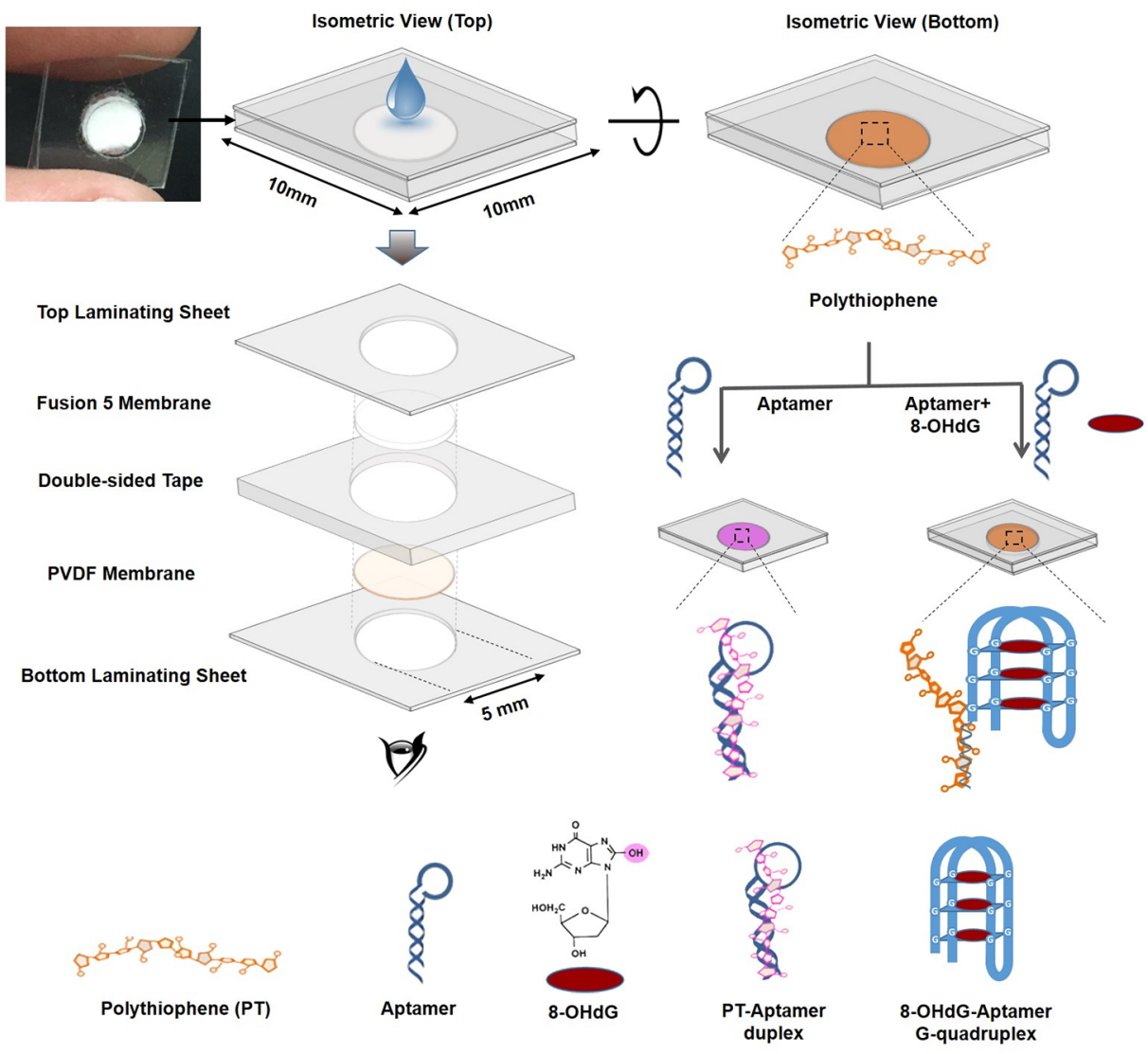

Figure 1. Schematic representation of the luminescent paper device illustrating distinguishable colorimetric responses of poly(3-alkoxy-4methylthiophene) (PT) for the G-quadruplex-secondary structure of the aptamer in the presence of 8-OHdG and for the PT-aptamer duplex in the absence of 8-OHdG.

spectroscopy, ${ }^{22,23}$ fluorescence spectroscopy, ${ }^{24,25}$ resonance light scattering, ${ }^{26}$ and enzyme-linked immunosorbent assay ${ }^{27}$ have also been explored for the detection of 8 -OHdG. However, most of them are laboratory techniques requiring sophisticated instrumentation and trained personnel to perform the assay. Thus, it is highly desirable to develop a point-of-care (POC) assay for 8-OHdG. Over the recent years, the concept of paper-based sensing has gained significant attention for fabrication of lab-on-a-chip devices enabling POC analysis. ${ }^{28-32}$ Although paper-based biosensors for 8-OHdG analysis have been reported, most of the approaches involve antibodies, which pose stability concerns. ${ }^{33,34}$ In this context, a paper-based colorimetric assay for POC 8-OHdG assay utilizing aptamers is proposed.

The proposed device comprises a polyvinylidene fluoride (PVDF) membrane, a filter paper, and lamination sheets. Cationic polythiophene (PT), with triethylamine pendant groups, is utilized for colorimetric reporting because of its superior optical and electronic properties. ${ }^{35-37}$ Herein, PT is impregnated in the PVDF membrane along with an aptamer as the 8-OHdG recognition element. A guanine-rich aptamer sequence, reported to exhibit a high affinity for $8-\mathrm{OHdG}$, is utilized in this study. ${ }^{38}$ As illustrated in Figure 1, the sensing strategy is based on monitoring the changes in optical properties of PT associated with its conformational changes upon interactions with the aptamer in the presence and in the absence of 8-OHdG, respectively. Complexation of PTaptamer in the PVDF membrane produces a duplex because of the electrostatic interactions between PT and the aptamer.
These interactions induce fluorescence quenching, resulting in a color transition from orange (PT) to purple (PT-aptamer). The color transition of PT is not observed in the presence of 8$\mathrm{OHdG}$, as complexation is perturbed, yielding fluorescent PTaptamer-8-OHdG complexes, owing to the affinity between the aptamer and 8-OHdG. Therefore, the orange color (PTaptamer-8-OHdG) and the significantly different purple color (PT-aptamer) correspond to the presence and the absence of 8 -OHdG, respectively, thereby enabling the naked eye detection of $8-\mathrm{OHdG}$. The assay of $8-\mathrm{OHdG}$ yielded linear fluorometric and colorimetric responses for clinically relevant concentration ranges between $50 \mathrm{pM}$ and $500 \mathrm{nM}$ with limits of detection of $\sim 300 \mathrm{pM}$ and $\sim 350 \mathrm{pM}$, respectively, enabling POC detection without requiring sophisticated instrumentation.

\section{EXPERIMENTAL SECTION}

Materials and Chemicals. 8-OHdG, artificial (synthetic) urine, and all the reagents and solvents were obtained from Sigma-Aldrich and used without further purification. The 8-OHdG aptamer (sequence, 5'-GCG GGC GAT CGG CGG GGG GTG CGT GCG CTC TGT GCC AGG GGG TGG GAC AGA TCA TAT GGG GGT GCT $\left.-3^{\prime}\right)^{38}$ was synthesized by IDT, Singapore, followed by PAGE purification. PVDF centrifugation tubes of $0.1 \mu \mathrm{m}$ pore size were purchased from Merck. Fusion 5 membranes made of glass fibers were purchased from Whatman and used for the pretreatment of samples.

Synthesis, Sample Preparation, and Analysis. A water-soluble PT was synthesized by oxidative polymerization of its monomer $(\mathrm{m} 1)$ using the protocol reported previously. ${ }^{39}$ The protocol adopted for the synthesis of $\mathrm{m} 1$ and $\mathrm{PT}$ and their corresponding NMR spectra are 
(a)

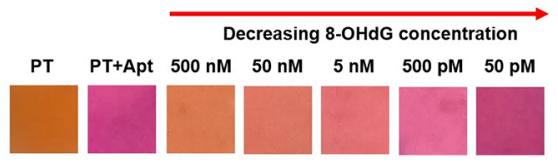

(c)
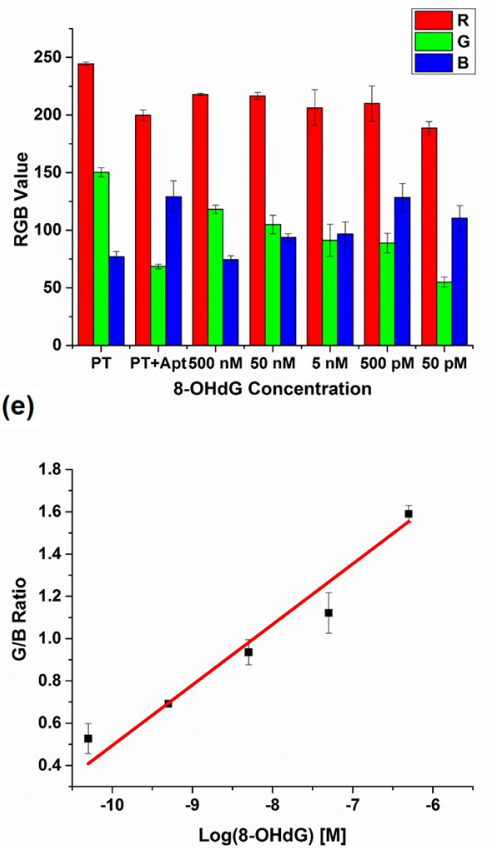

(b)

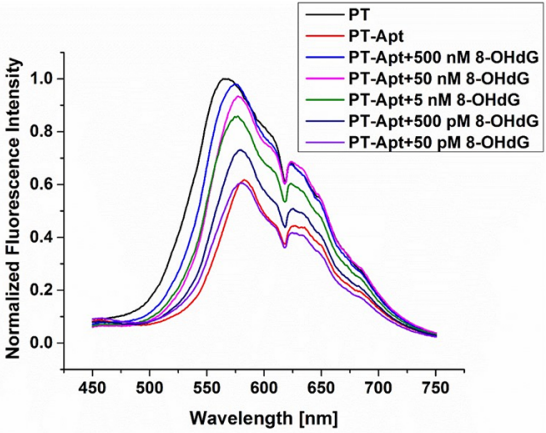

(d)

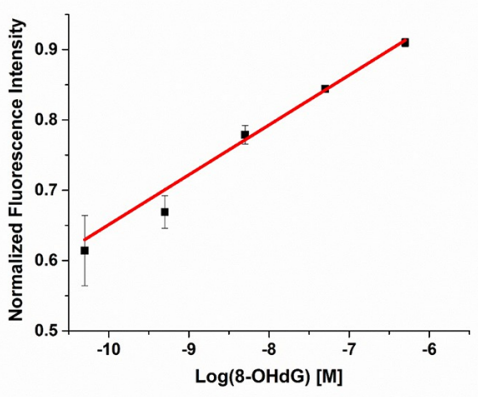

(f)

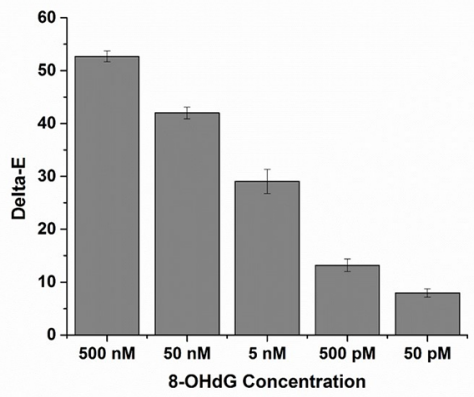

Figure 2. (a) Color-coded digital array of PT with the aptamer and various 8-OHdG concentrations. (b) Normalized fluorescence spectra upon addition of the aptamer and various concentrations of 8 -OHdG (c) mean RGB values and standard deviation for $n=3$ (d) semilogarithmic plot of the 8-OHdG concentration versus normalized fluorescence intensity and the corresponding linear fit (e) semilogarithmic plot of the 8-OHdG concentration vs ratio of $\mathrm{G} / \mathrm{B}$ values and the corresponding linear fit (f) mean $\Delta E$ values and standard deviation for $n=3$ of various 8 -OHdG concentrations with respect to $\mathrm{PT}$-aptamer.

provided in Supporting Information Figure S1. NMR spectra were recorded using a Bruker ADVANCE $400 \mathrm{MHz}$ spectrometer. In brief, $200 \mu \mathrm{L}$ of $5 \mu \mathrm{M}$ PT was added to a centrifugation tube. Tubes were centrifuged at $8000 \mathrm{rpm}$ for $3 \mathrm{~min}$. The abovementioned step was repeated three more times. After impregnation of PT, the PVDF membranes were washed twice using DI water at $8000 \mathrm{rpm}$ for $3 \mathrm{~min}$ and dried overnight. PVDF membranes deposited with PT were then peeled off from the centrifugation tube for device fabrication.

A vertical flow-through configuration was adopted for its advantages over the lateral-flow format in terms of the low sample volume and decreased assay time. Capillary or passive forces allow this system to operate without the requirement of an external pump. ${ }^{40}$ The device consisted of two laminated sheets containing a Fusion 5 filter paper and a PVDF membrane impregnated with PT for colorimetric sensing (Figure 1). The lamination sheets containing Fusion 5 and PVDF membrane were then attached using a double-sided adhesive tape.

A PVDF membrane impregnated with PT was then incubated with a $1 \mu \mathrm{M}$ aptamer followed by the addition of varying concentrations of 8 -OHdG $(\sim 5 \mu \mathrm{L})$. To ascertain the obtained color changes, the devices were placed on a UV illumination zone (portable UV lamp, at $365 \mathrm{~nm}, 8 \mathrm{~W}$ ), and digital images were captured using a Sony Xperia $\mathrm{Z} 5$ premium mobile phone camera positioned at a fixed distance from the device. Subsequently, the digital images were transferred to a computer as jpeg files for image processing. The raw images were imported into "ImageJ" software, and the centroids of paper membranes $(75 \times 75$ pixels $)$ were utilized to create a digital array. The RGB values were evaluated using ImageJ software to ascertain colorimetric responses. The process of creating a color-coded digital array is shown in Figure S2. Fluorescence measurements were performed using an Avantes spectrofluorometer, and the emission spectra were recorded for analysis.

\section{RESULTS AND DISCUSSION}

Interaction of PT with the Aptamer and 8-OHdG. The assay for the naked eye detection of $8-\mathrm{OHdG}$ is based on monitoring the changes in optical properties of PT upon complexation with the aptamer in the presence and in the absence of 8-OHdG. The aptamer utilized for affinity binding to 8 -OHdG with a $K_{\mathrm{d}}$ value of $0.1 \mu \mathrm{mol} \mathrm{L}{ }^{-138}$ was modified by removing primer 1 and primer 2 sequences at $3^{\prime}$ and $5^{\prime}$ to serve as an effective recognition element for proposed assay. PT (5 $\mu \mathrm{M}$ ) and $1 \mu \mathrm{M}$ aptamer (in DI water) were determined to be the optimum concentrations for monitoring 8-OHdG at clinically relevant concentration levels ( $\mathrm{pM}$ to $\mathrm{nM}$ ). Figure 2a illustrates that the addition of the $1 \mu \mathrm{M}$ aptamer onto the PTimpregnated PVDF membrane yields a color change from 
(a)

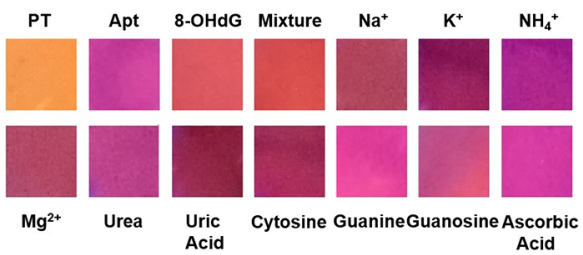

(b)

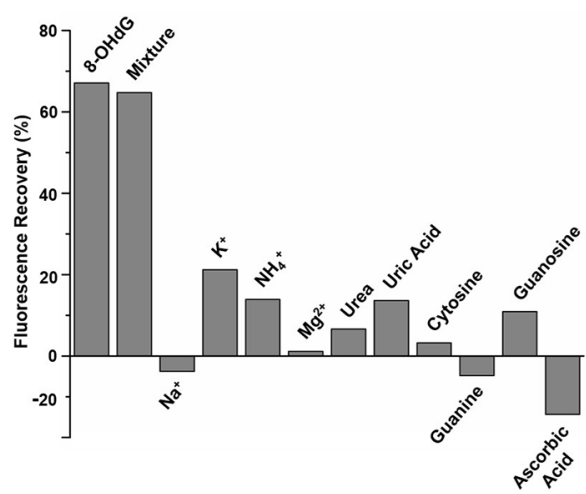

Figure 3. (a) Color-coded digital array of the proposed platform with 8-OHdG and interfering molecules. (b) Fluorescence recovery upon the addition of 8-OHdG, metal ions, 8-OHdG analogues, and other potentially interfering compounds (mixture: 8-OHdG with interfering compounds such as uric acid, cytosine, guanine, guanosine, and ascorbic acid).

orange (PT on the PVDF membrane) to purple (PT-aptamer complex on the PVDF membrane). The duplex formation is associated with backbone planarization and aggregation of the PT because of the electrostatic interactions between the positively charged PT (associated with the triethylamine groups) and the negatively charged aptamer. These electrostatic interactions cause a change from a random-coiled morphology of PT to a planar $\pi$-stacking morphology, leading to a substantial quenching of the fluorescence signal and a concomitant color transition. A saturated colorimetric response for 8-OHdG is expected to overlap with the color of PT, provided there is complete $8-\mathrm{OHdG}$-aptamer complex formation and consequent weakening of electrostatic interactions between PT and the aptamer, whereas assay concentrations would yield intermediate colorimetric responses depending on the strength of the interaction between the PT and the aptamer. Therefore, for the assay, different concentrations of $8-\mathrm{OHdG}$ from $50 \mathrm{pM}$ to $500 \mathrm{nM}$ were added to the PT-impregnated PVDF membranes containing the aptamer. 8OHdG induces a conformational change of the aptamer containing a guanine-rich nucleic acid sequence to form Gquadruplex structures as shown in Figure 1. The formation of the rigid structure of G-quadruplex reduces the electrostatic interactions between the PT and the aptamer, thereby leading to fluorescence and color recovery of PT. Figure 2a,b illustrates that both color change and fluorescence recovery occur with the increasing concentration of $8-\mathrm{OHdG}$, which is attributed to the formation of a G-quadruplex structures. The color changes from orange to purple upon decreasing the 8-OHdG concentrations, as shown in Figure 2a. The two significantly different optical signals (PT-aptamer and PT-aptamer-8$\mathrm{OHdG}$ ) are subsequently analyzed and correlated to the concentration of 8 -OHdG. Figure 2 a shows a $75 \times 75$ pixel color-coded digital array of various concentrations of 8-OHdG (500 $\mathrm{nM}$ to $50 \mathrm{pM}$, left to right) for subsequent analysis.

In order to validate the assay, fluorescence spectra were recorded for the PT-aptamer-incorporated PVDF membranes for various concentrations of 8-OHdG. Upon excitation at 420 $\mathrm{nm}$, an emission maxima centered at $566 \mathrm{~nm}$ with a shoulder at around $625 \mathrm{~nm}$ appeared, as shown in Figure $2 \mathrm{~b}$. The spectrum of the PT-impregnated membrane is red-shifted (about $25 \mathrm{~nm}$ ) with respect to the solution spectrum, likely because of partial self-aggregation/confinement of PT. ${ }^{35,41,42}$ Even though aggregation causes loss in fluorescence intensity, the PTimpregnated membrane shows clearly visible orange color emission upon UV exposure (365 nm), as illustrated in Figure 2a. Moreover, steady-state fluorescence spectroscopy reveals substantial quenching in emission and red shift (about $15 \mathrm{~nm}$ ) upon the addition of the aptamer (black to red), indicating a duplex formation between PT and the aptamer. As observed from Figure $2 b$, the fluorescence intensity gradually increases with the increasing concentration of 8-OHdG because of the formation of 8-OHdG-aptamer G-quadruplex complexes. Further evidence of complex formation is obtained from the RGB analysis of the color-coded digital array (Figure 2c) via ImageJ software. As shown in Figure $2 \mathrm{c}$, there is a substantial change in the G (from 150.3 to 68.4) and B (from 76.9 to 129.1) values upon the addition of the aptamer to PT, whereas upon the addition of $500 \mathrm{nM}$ 8-OHdG with the aptamer, the G (118.2) and B (74.5) values revert back to that of PT, indicating almost complete recovery of color. It could be observed that the G (from 118.2 to 63 ) and B (from 74.5 to 119.7) values decrease and increase, respectively, with the decreasing concentrations of 8-OHdG. To ensure reproducibility, spectral and RGB analysis of the impregnated membrane were conducted for three independent assays. Figure $2 \mathrm{c}$ shows that RGB analysis is in agreement with the naked eye observation (Figure 2a). The correlation between the 8OHdG concentration and the normalized fluorescence intensity is evaluated using the ratio $I / I_{\mathrm{PT}}$, where $I$ and $I_{\mathrm{PT}}$ represent the integrated fluorescence intensity from 450 to $750 \mathrm{~nm}$ for the assay and PT, respectively. The linearity of the response was analyzed using a semilogarithmic plot of the normalized fluorescence intensity versus concentration of 8-OHdG. Figure $2 \mathrm{~d}$ shows the linear regression equation $(Y=0.0767 \log X+$ $\left.1.3998, R^{2}=0.989, n=3\right)$, yielding a limit of detection of $\sim 300$ $\mathrm{pM}(\mathrm{S} / \mathrm{N}=3)$.

Similarly, the correlation between the concentration of 8OHdG and the colorimetric response is evaluated using the $G$ and $B$ values from the Figure $2 c$. The linearity of the response was ascertained by a semilogarithmic plot of $G / B$ ratio versus the 8-OHdG concentration $(n=3)$, as shown in Figure 2e with a linear regression equation of $\left(Y=0.2557 \log X+3.0955, R^{2}=\right.$ $0.96, n=3)$, yielding a limit of detection of $\sim 350 \mathrm{pM}(\mathrm{S} / \mathrm{N}=$ 3 ). Furthermore, $\Delta E$ values, ranging between 0 and 100, are a metric for human eye perception of color differences and are calculated using the International Commission of Illumination (CIE) 1976 algorithm. ${ }^{43}$ Figure $2 \mathrm{f}$ illustrates a broad range of $\Delta E$ ( $\sim$ from 8 to 53 ) for PT-aptamer-8-OHdG and PTaptamer in the assay concentration range of $8-\mathrm{OHdG}(50 \mathrm{pM}$ 
(a)

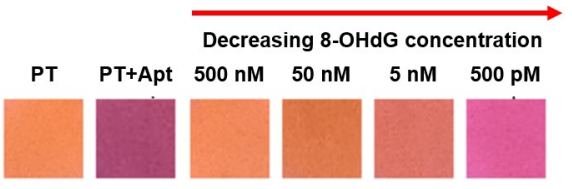

(b)

(c)
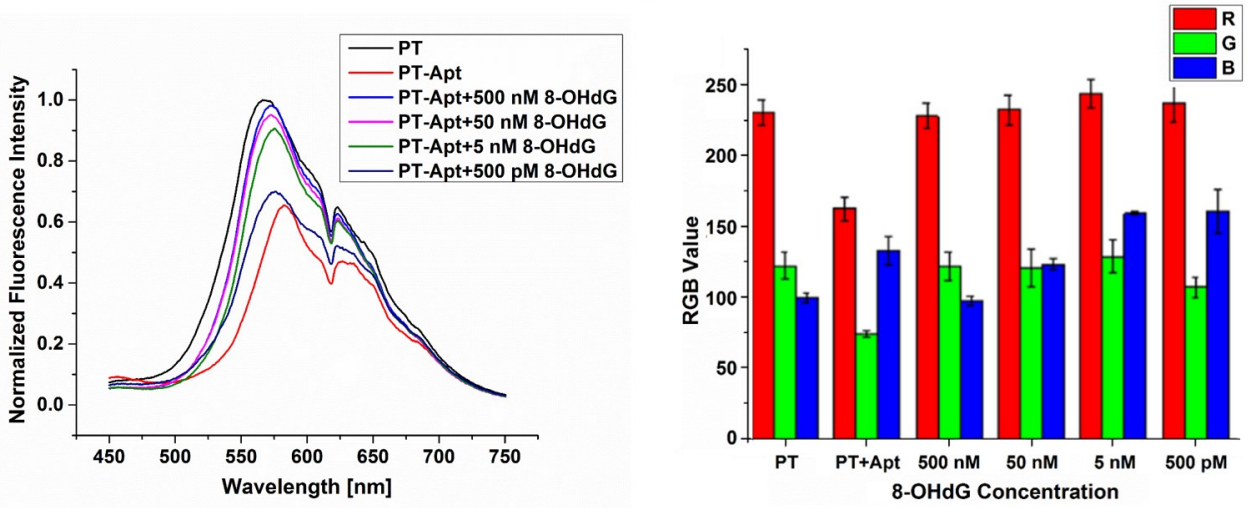

Figure 4. (a) Color-coded digital array of PT with the aptamer and various 8-OHdG concentrations in artificial urine. (b) Normalized fluorescence spectra upon the addition of the aptamer and various 8-OHdG concentrations. (c) Mean RGB values and standard deviation for $n=3$.

to $500 \mathrm{nM}$ ), indicating that the color difference between PTaptamer-8-OHdG and PT-aptamer could be visually distinguished.

Selectivity Studies. The selectivity of the platform was evaluated using metal ions, potentially interfering compounds, and other 8-OHdG analogues that may coexist in urine. As observed from Figure $3 \mathrm{a}$, the metal ions, analogues, and the interfering compounds yield almost similar visual responses (varying shades of purple) as that of the aptamer, whereas 50 $\mathrm{nM}$ 8-OHdG with and without interfering molecules shows a recovery of color to orange from purple. Figure $3 \mathrm{~b}$ shows the fluorescence recovery of $8-\mathrm{OHdG}$, metal ions, and other interfering compounds. The fluorescence recovery was calculated as $\left(I-I_{\text {aptamer }}\right) /\left(I_{\mathrm{PT}}-I_{\text {aptamer }}\right)$, where $I_{\text {aptamer }}$ represents the integrated fluorescence intensity of the PTaptamer complex from 450 to $750 \mathrm{~nm}$. Small to negligible fluorescence recovery is observed upon the addition of 8OHdG analogues and metal ions, even at 100 times higher concentration than that of $8-\mathrm{OHdG}$, as shown in Figure $3 \mathrm{~b}$. The higher concentrations of ions such as potassium and 8OHdG analogues interfere with the detection of 8 -OHdG (maximum of $\sim 20 \%$ for potassium), as observed from Figure $3 \mathrm{~b}$, suggesting that these molecules could also induce a conformational change of the aptamer. ${ }^{22}$ However, such ions could be eliminated from the sample matrices by dialysis using a dialysis bag with a molecular weight cutoff of $200 \mathrm{Da}^{22}$ In the mixture of $50 \mathrm{nM} 8-\mathrm{OHdG}$ with $8-\mathrm{OHdG}$ analogues and interfering compounds such as guanosine, guanine, uric acid, ascorbic acid, and cytosine, a recovery of around $64 \%$ was observed, as against $67 \%$ for $50 \mathrm{nM} 8-\mathrm{OHdG}$ without interfering compounds, illustrating excellent selectivity of the proposed methodology.

Analysis in Artificial Urine. To validate the proposed methodology for clinical applications, different concentrations of $8-\mathrm{OHdG}$ were spiked in the synthetic urine and tested. As shown in Figure 4a, varying colorimetric responses are obtained upon the addition of different concentrations of 8 -OHdG in spiked urine. The orange PT color appears relatively darker upon the addition of artificial urine samples without the aptamer, and there is a reduction of the fluorescence intensity, as compared to that of DI water. A possible explanation is that other anions in artificial urine might contribute to the formation of planar $\pi$-stacking morphology upon electrostatic interactions with PT and thereby lowering the PT fluorescence. However, as shown in Figure 4a, the color changes to purple upon the addition of the aptamer, which is in agreement with the responses in DI.

A color change from orange to pink was observed upon decreasing the concentration of $8-\mathrm{OHdG}$ from $500 \mathrm{nM}$ to 500 pM (left to right in Figure 4a). As shown in Figure 4b, there is also a substantial quenching of fluorescence upon the addition of the aptamer (black to red), and the fluorescence intensity recovers gradually with increasing the $8-\mathrm{OHdG}$ concentration (from $500 \mathrm{pM}$ to $500 \mathrm{nM}$ ). Further evidence of 8-OHdG aptamer complex formation is obtained from the RGB analysis, as shown in Figure. 4c. Upon the addition of the aptamer, there is a significant change of RGB values from PT to PT-aptamer (orange to purple), whereas upon the addition of $500 \mathrm{nM} \mathrm{8-}$ OHdG, the RGB values almost revert back to PT, indicating the change in color from purple to orange. Changes in RGB values were also observed with varying concentrations of 8OHdG. Figure 4c shows that RGB analysis is in agreement with the naked eye observation, and the proposed methodology can be used for the detection of $8-\mathrm{OHdG}$ at clinically relevant concentration ranges in urine samples. Although the estimated LOD from Figure 4 is around $500 \mathrm{pM}$, it should be noted that the responses were recorded using synthetic urine at a $\mathrm{pH}$ of 6.7 and at temperatures ranging between 10 and $40{ }^{\circ} \mathrm{C}$. The influence of temperature, $\mathrm{pH}$, and light shown in Figures S3 and $\mathrm{S} 4$ suggests that acidic $\mathrm{pH}(<6)$ might cause interference in the sensor response and that the samples should be stored in dark. Operation of paper-based assay is not recommended above $40{ }^{\circ} \mathrm{C}$.

Comparison with Other Sensing Strategies. Electrochemical methodologies such as CV and DPV for quantitative analysis of 8 -OHdG have claimed subnanomolar detection 
sensitivities. ${ }^{17,20}$ Similarly, spectroscopic methods such as ultraviolet-visible spectroscopy (UV-vis) and fluorescence spectroscopy have also been explored for the detection of 8$\mathrm{OHdG}^{22-24}$ with subnanomolar and picomolar sensitivities. However, most of the reported methodologies involve tedious and sophisticated assay protocols. Table 1 illustrates that the

Table 1. Comparison of Various Sensing Platforms for the Detection of 8-OHdG

\begin{tabular}{llll}
\multicolumn{1}{c}{ methodology } & detection range & limit of detection & refs \\
LSV & $0.7-70 \mu \mathrm{M}$ & $100 \mathrm{nM}$ & 21 \\
DPV & $0.5-35 \mu \mathrm{M}$ & $31.3 \mathrm{nM}$ & 20 \\
$\mathrm{CV}$ & $0.0563-16.4 \mu \mathrm{M}$ & $18.8 \mathrm{nM}$ & 19 \\
$\mathrm{CV}$ & $5.6-1155 \mu \mathrm{M}$ & $0.875 \mathrm{nM}$ & 17 \\
CE-ECD & $0.01-1.5 \mu \mathrm{M}$ & $2.6 \mathrm{nM}$ & 16 \\
UV-vis & $5.6-282 \mathrm{nM}$ & $1.7 \mathrm{nM}$ & 22 \\
UV-vis & $0.466-247 \mathrm{nM}$ & $141 \mathrm{pM}$ & 23 \\
colorimetric and & $35.3-706.1 \mathrm{nM}$ & $7.3 \mathrm{nM}$ & 34 \\
$\quad$ electrochemical & & & \\
fluorescence & $3.96-211 \mathrm{nM}$ & $1.19 \mathrm{nM}$ & 24 \\
colorimetric and & $0.5-500 \mathrm{nM}$ & $\sim 350 \mathrm{pM}$ & reported \\
fluorescence & & $\begin{array}{l}\text { colorimetric } \\
\text { and } \sim 300 \mathrm{pM}\end{array}$ & methodology \\
& & fluorescence & \\
\hline
\end{tabular}

limit of detection of the proposed methodology is either better or comparable to previous reports, while exhibiting a wider linear dynamic range for $8-\mathrm{OHdG}$ detection. Our findings indicate a linear response for both color and the fluorescence intensity for clinically relevant concentration ranges of $8-\mathrm{OHdG}$ $(50 \mathrm{pM} \text { to } 500 \mathrm{nM})^{13}$ with limits of detection of $\sim 350$ and 300 pM, respectively.

In addition, the overall detection processes are conducted at ambient conditions, and the experimental procedure is facile. Furthermore, the proposed methodology possesses significant advantages over conventional sensing platforms such as ease of use, ease of fabrication, and low cost with an assay time of 30 min.

\section{CONCLUSIONS}

A disposable paper-based analytical device for the naked eye detection of oxidative stress biomarker is reported. The proposed methodology has been validated for 8-OHdG assay using luminescent reporter (PT)-impregnated PVDF membranes. The obtained colorimetric responses in artificial urine indicate the potential use in clinical applications for early diagnosis of various diseases associated with ROS. The fabricated platform could be ideal for POC applications and for the detection of oxidative stress at locations where sophisticated instrumentation is sparse or even nonexistent. Future studies will emphasize on the detection of $8-\mathrm{OHdG}$ in patient samples, optimization of device storage conditions, development of assay for other potential ROS markers, and bench marking with established methodologies.

\section{ASSOCIATED CONTENT}

\section{S Supporting Information}

The Supporting Information is available free of charge on the ACS Publications website at DOI: 10.1021/acsami.7b17252.

Synthesis protocol and the NMR spectra for the precursors/monomer and $\mathrm{PT}$, color-coded array creation process for RGB analysis, influence of temperature and
$\mathrm{pH}$ on 8-OHdG assay, and influence of light, degree of polymerization, and GMP on PT (PDF)

\section{AUTHOR INFORMATION}

\section{Corresponding Authors}

*E-mail: alps@ntu.edu.sg (A.P.).

*E-mail: bliedberg@ntu.edu.sg (B.L.).

ORCID

Bo Liedberg: 0000-0003-2883-6953

Notes

The authors declare no competing financial interest.

\section{ACKNOWLEDGMENTS}

The authors wish to acknowledge funding support from Tier 1 MOE-RG 82/12, and NITHM M4081989.070.500000 research grants. The authors further wish to thank Profs. Ali Yusuf and Bernhard Boehm, LKC School of Medicine, Nanyang Technological University, for fruitful discussions.

\section{REFERENCES}

(1) Semchyshyn, H. M.; Lushchak, V. I. Interplay Between Oxidative and Carbonyl Stresses: Molecular Mechanisms, Biological Effects and Therapeutic Strategies of Protection. Oxidative Stress-Molecular Mechanisms and Biological Effects; InTech, 2012.

(2) Kivatinitz, S. C. Relationship Between Protein Oxidation Markers and Oxidative Stress Biomarkers, Inflammatory Diseases. Immunopathology, Clinical and Pharmacological Bases; InTech, 2012.

(3) Dalle-Donne, I.; Giustarini, D.; Colombo, R.; Rossi, R.; Milzani, A. Protein Carbonylation in Human Diseases. Trends Mol. Med. 2003, 9, $169-176$.

(4) Niedworok, J.; Fijałkowski, P. Effect of Long-Term Aluminium Chloride Intoxication on Selected Biochemical Parameters and Oxidative-Antioxidative Balance in Experimental Animals. Pol. J. Environ. Stud. 2004, 13, 41-43.

(5) Lowe, F. Biomarkers of Oxidative Stress. Systems Biology of Free Radicals and Antioxidants; Springer, 2014; pp 65-87.

(6) Dalle-Donne, I.; Rossi, R.; Colombo, R.; Giustarini, D.; Milzani, A. Biomarkers of Oxidative Damage in Human Disease. Clin. Chem. 2006, 52, 601-623.

(7) Valavanidis, A.; Vlachogianni, T.; Fiotakis, C. 8-Hydroxy-2'Deoxyguanosine (8-OHdG): A Critical Biomarker of Oxidative Stress and Carcinogenesis. J. Environ. Sci. Health, Part C: Environ. Carcinog. Ecotoxicol. Rev. 2009, 27, 120-139.

(8) Kasai, H. Analysis of a form of Oxidative DNA Damage, 8Hydroxy-2'-Deoxyguanosine, As a Marker of Cellular Oxidative Stress During Carcinogenesis. Mutat. Res., Rev. Mutat. Res. 1997, 387, 147163.

(9) Subash, P.; Gurumurthy, P.; Sarasabharathi, A.; Cherian, K. M. Urinary 8-OHdG: A Marker of Oxidative Stress to DNA and Total Antioxidant Status in Essential Hypertension with South Indian Population. Indian J. Clin. Biochem. 2010, 25, 127-132.

(10) Guan, Y.; Zhou, G.; Ye, J. Fast Quantification of Salivary 8Hydroxy-2'-Deoxyguanosine as DNA Damage Biomarker Using CE with Electrochemical Detection. Chromatographia 2014, 77, 603-607.

(11) Bolner, A.; Pilleri, M.; De Riva, V.; Nordera, G. Plasma and Urinary HPLC-ED Determination of the Ratio of 8-OHdG/2-dG in Parkinson's Disease. Clin. Lab. 2011, 57, 859.

(12) Arnett, S. D.; Osbourn, D. M.; Moore, K. D.; Vandaveer, S. S.; Lunte, C. E. Determination of 8-Oxoguanine and 8-Hydroxy-2'Deoxyguanosine in the Rat Cerebral Cortex Using Microdialysis Sampling and Capillary Electrophoresis with Electrochemical Detection. J. Chromatogr. B: Anal. Technol. Biomed. Life Sci. 2005, $827,16-25$.

(13) Cutler, R. G.; Rodriguez, H. Critical Reviews of Oxidative Stress and Aging: Advances in Basic Science, Diagnostics and Intervention; World Scientific, 2003; Vol. 2. 
(14) Koide, S.; Kinoshita, Y.; Ito, N.; Kimura, J.; Yokoyama, K.; Karube, I. Determination of Human Serum 8-hydroxy-2'-deoxyguanosine (8-OHdG) by HPLC-ECD Combined with Solid Phase Extraction (SPE). J. Chromatogr. B: Anal. Technol. Biomed. Life Sci. 2010, 878, 2163-2167.

(15) Weimann, A.; Belling, D.; Poulsen, H. E. Quantification of 8Oxo-Guanine and Guanine as the Nucleobase, Nucleoside and Deoxynucleoside Forms in Human Urine by High-Performance Liquid Chromatography-Electrospray Tandem Mass Spectrometry. Nucleic Acids Res. 2002, 30, 7e-7.

(16) Zhang, S. W.; Zou, C. J.; Luo, N.; Weng, Q. F.; Cai, L. S.; Wu, C. Y.; Xing, J. Determination of Urinary 8-Hydroxy-2'-Deoxyguanosine by Capillary Electrophoresis with Molecularly Imprinted Monolith in-Tube Solid Phase Microextraction. Chin. Chem. Lett. 2010, 21, 85-88.

(17) Jia, L.-P.; Liu, J.-F.; Wang, H.-S. Electrochemical Performance and Detection of 8-Hydroxy-2'-Deoxyguanosine at Single-Stranded DNA Functionalized Graphene Modified Glassy Carbon Electrode. Biosens. Bioelectron. 2015, 67, 139-145.

(18) Gutiérrez, A.; Gutiérrez, S.; García, G.; Galicia, L.; Rivas, G. A. Determinatiom of 8-Hydroxy 2'-Deoxyguanosine Using Electrodes Modified with a Dispersion of Carbon Nanotubes in Polyethylenimine. Electroanalysis 2011, 23, 1221-1228.

(19) Guo, Z.; Liu, X.; Liu, Y.; Wu, G.; Lu, X. Constructing a Novel 8Hydroxy-2'-Deoxyguanosine Electrochemical Sensor and Application in Evaluating the Oxidative Damages of DNA and Guanine. Biosens. Bioelectron. 2016, 86, 671-676.

(20) Sun, X.; Zhang, L.; Zhang, H.; Qian, H.; Ji, J.; Tang, L.; Li, Z.; Zhang, G. Electrochemical detection of 8-Hydroxy-2'-Deoxyguanosine as a Biomarker for Oxidative DNA Damage in HEK293 Cells Exposed to 3-Chloro-1, 2-Propanediol. Anal. Methods 2015, 7, 6664-6671.

(21) Li, T.-H.; Jia, W.-L.; Wang, H.-S.; Liu, R.-M. Electrochemical Performance Of 8-Hydroxy-2'-Deoxyguanosine and its Detection at Poly (3-methylthiophene) Modified Glassy Carbon Electrode. Biosens. Bioelectron. 2007, 22, 1245-1250.

(22) Wang, J.-C.; Wang, Y.-S.; Rang, W.-Q.; Xue, J.-H.; Zhou, B.; Liu, L.; Qian, Q.-M.; Wang, Y.-S.; Yin, J.-C. Colorimetric Determination of 8-Hydroxy-2'-Deoxyguanosine Using Label-Free Aptamer and Unmodified Gold Nanoparticles. Microchim. Acta 2014, 181, 903-910.

(23) Liu, H.; Wang, Y.-S.; Wang, J.-C.; Xue, J.-H.; Zhou, B.; Zhao, H.; Liu, S.-D.; Tang, X.; Chen, S.-H.; Li, M.-H.; Cao, J.-X. A Colorimetric Aptasensor for the Highly Sensitive Detection of 8-Hydroxy-2'Deoxyguanosine Based on G-Quadruplex-Hemin Dnazyme. Anal. Biochem. 2014, 458, 4-10.

(24) Liu, H.; Wang, Y.-S.; Tang, X.; Yang, H.-X.; Chen, S.-H.; Zhao, H.; Liu, S.-D.; Zhu, Y.-F.; Wang, X.-F.; Huang, Y.-Q. A Novel Fluorescence Aptasensor for 8-Hydroxy-2'-Deoxyguanosine Based on the Conformational Switching of $\mathrm{K}^{+}$-Stabilized G-Quadruplex. J. Pharm. Biomed. Anal. 2016, 118, 177-182.

(25) Dou, K.; Chen, G.; Yu, F.; Liu, Y.; Chen, L.; Cao, Z.; Chen, T.; Li, Y.; You, J. Bright and Sensitive Ratiometric Fluorescent Probe Enabling Endogenous FA Imaging and Mechanistic Exploration of Indirect Oxidative Damage due to FA In Various Living Systems. Chem. Sci. 2017, 8, 7851-7861.

(26) Wang, J.-C.; Wang, Y.-S.; Xue, J.-H.; Zhou, B.; Qian, Q.-M.; Wang, Y.-S.; Yin, J.-C.; Zhao, H.; Liu, H.; Liu, S.-D. An Ultrasensitive Label-Free Assay of 8-Hydroxy-2'-Deoxyguanosine Based on the Conformational Switching of Aptamer. Biosens. Bioelectron. 2014, 58, $22-26$.

(27) Wu, L. L.; Chiou, C.-C.; Chang, P.-Y.; Wu, J. T. Urinary 8OHdG: A Marker of Oxidative Stress to DNA and a Risk Factor for Cancer, Atherosclerosis And Diabetics. Clin. Chim. Acta 2004, 339, 19.

(28) Fu, X.; Cheng, Z.; Yu, J.; Choo, P.; Chen, L.; Choo, J. A SERSBased Lateral Flow Assay Biosensor for Highly Sensitive Detection of HIV-1 DNA. Biosens. Bioelectron. 2016, 78, 530-537.

(29) Sun, M.; Johnson, M. A. Measurement of Total Antioxidant Capacity in sub- $\mu$ l Blood Samples Using Craft Paper-Based Analytical Devices. RSC Adv. 2015, 5, 55633-55639.
(30) Dungchai, W.; Sameenoi, Y.; Chailapakul, O.; Volckens, J.; Henry, C. S. Determination of Aerosol Oxidative Activity Using Silver Nanoparticle Aggregation on Paper-Based Analytical Devices. Analyst 2013, 138, 6766-6773.

(31) Liana, D. D.; Raguse, B.; Gooding, J. J.; Chow, E. Recent Advances in Paper-Based Sensors. Sensors 2012, 12, 11505-11526.

(32) Parolo, C.; Merkoçi, A. Paper-Based Nanobiosensors for Diagnostics. Chem. Soc. Rev. 2013, 42, 450-457.

(33) Martins, G. V.; Tavares, A. P.; Fortunato, E.; Sales, M. G. F. Paper-Based Sensing Device for Electrochemical Detection of Oxidative Stress Biomarker 8-Hydroxy-2'-deoxyguanosine (8-OHdG) in Point-of-Care. Sci. Rep. 2017, 7, 14558.

(34) Zhu, X.; Shah, P.; Stoff, S.; Liu, H.; Li, C.-z. A Paper Electrode Integrated Lateral Flow Immunosensor for Quantitative Analysis of Oxidative Stress Induced DNA Damage. Analyst 2014, 139, 28502857.

(35) Ho, H.-A.; Najari, A.; Leclerc, M. Optical Detection of DNA and Proteins with Cationic Polythiophenes. Acc. Chem. Res. 2008, 41, 168178.

(36) Lee, K.; Povlich, L. K.; Kim, J. Recent Advances in Fluorescent and Colorimetric Conjugated Polymer-Based Biosensors. Analyst 2010, 135, 2179-2189.

(37) Zade, S. S.; Bendikov, M. Twisting of Conjugated Oligomers and Polymers: Case Study of Oligo-and Polythiophene. Chem.-Eur. J. 2007, 13, 3688-3700.

(38) Miyachi, Y.; Shimizu, N.; Ogino, C.; Fukuda, H.; Kondo, A. Selection of a DNA Aptamer that Binds 8-OHdg Using GMP-Agarose. Bioorg. Med. Chem. Lett. 2009, 19, 3619-3622.

(39) Rajwar, D.; Ammanath, G.; Cheema, J. A.; Palaniappan, A.; Yildiz, U. H.; Liedberg, B. Tailoring Conformation-Induced Chromism of Polythiophene Copolymers for Nucleic Acid Assay at Resource Limited Settings. ACS Appl. Mater. Interfaces 2016, 8, 8349-8357.

(40) Vella, S. J.; Beattie, P.; Cademartiri, R.; Laromaine, A.; Martinez, A. W.; Phillips, S. T.; Mirica, K. A.; Whitesides, G. M. Measuring Markers of Liver Function Using a Micropatterned Paper Device Designed for Blood from a Fingerstick. Anal. Chem. 2012, 84, 28832891.

(41) Yildiz, U. H.; Alagappan, P.; Liedberg, B. Naked Eye Detection of Lung Cancer Associated miRNA by Paper Based Biosensing Platform. Anal. Chem. 2012, 85, 820-824.

(42) Ho, H.-A.; Leclerc, M. Optical Sensors Based on Hybrid Aptamer/Conjugated Polymer Complexes. J. Am. Chem. Soc. 2004, 126, 1384-1387.

(43) Hill, B.; Roger, T.; Vorhagen, F. W. Comparative Analysis of the Quantization of Color Spaces on the Basis of the CIELAB ColorDifference Formula. ACM Trans. Graph. 1997, 16, 109-154. 